\title{
A NEAR FIELD SPHERICAL WAVE INVERSE SYNTHETIC APERTURE RADAR TECHNIQUE
}

A.Broquetas *, L. Jofre, A.Cardama

Dept. Teoria del Senyal i Comunicacions, Grup AMR

ETSE Telecomunicació - UPC

P.O.Box 30002, 08080 Barcelona, Spain

\section{Introduction}

Conventional Inverse Synthetic Aperture Radar techniques can form radar images of targets by processing coherent RCS data obtained in a frequency range and angular scan $[1,2]$. Indoor RCS measurements are usually based on compact ranges, which are costly installations and present problems of edge difraction and surface tolerances of the antennas involved. In this paper a new imaging algorithm based on a near field spherical wave illumination is proposed. An spherical wave can be easily generated in an anechoic chamber using electrically small and low-cost horn antennas; no reflectors are used avoiding surface tolerance problems and diffraction can be minimized with an appropiate horn design. This paper presents an imaging near-field Spherical Wave ISAR (SWISAR) algorithm based on a focusing operator of the scattered fields on the target surface. Additional advantages of this algorithm are its ability to account for bistatic measurement geometries and lateral tapers in the spherical wave illumination.

Formulation

The mesurement geometry is shown in Fig.1. The target, assumed to be bidimensional, is placed on the Origin of the $(x, y)$ coordinate axis and can be rotated an angle $\theta$ with respect to the direction of the illuminating antenna. The target is modelled as a large group of independent point scatterers in the bandwith and directions of illumination of interest. This allows to define a 2D spatial reflectivity function $\Psi(x, y)$ in the exploration plane, which amplitude and phase characterizes each scatterer. Initially we will assume a monostatic measuremen geometry, in which the same isotropic antenna is used as emitter and receiver. The reconstruction of the image is an inverse scattering problem, where given the scattered fields, we wish to obtain a good approximation ' $\psi$ ' to the actual reflectivity $\psi$ of the target. An integral operator using the focusing function $\xi$ can be used on the measured scattered field $\mathrm{E}_{\mathrm{s}}$ as follows

$$
\psi^{\prime}(\rho, \phi)=\int_{0}^{\infty} \int_{0}^{2 \pi} E_{s}(f, \theta) \xi(f, \theta ; \rho, \phi) d \theta d f=\Psi(\rho, \phi)
$$

The focusing function adopted is 
$\xi(f, \theta ; \rho, \phi)=e^{j \frac{4 \pi}{\lambda} \sqrt{R^{2}+\rho^{2}-2 R \rho \cos (\phi-\theta)}}\left[R^{2}+\rho^{2}-2 R \rho \cos (\phi-\theta)\right] f(2)$

which compensates for the attenuation and phase of the scattering corresponding to each target point. If $\mathrm{R} \rightarrow \infty$ the reconstruction expression becomes an Inverse Fourier Transform expressed in polar coordinates, which is the inversion scheme commonly used in conventional plane wave ISAR. If a bistatic geometry is used with a receiver antenna at a distance $\mathrm{R} 2$ from the Origin and at angle $\beta$ with respect to the emitter antenna, the operator can be generalized as follows

$$
\begin{aligned}
& \xi(f, \theta ; \rho, \phi)=e^{j \frac{2 \pi}{\lambda} \sqrt{R^{2}+\rho^{2}-2 R \rho \cos (\phi-\theta)}} \sqrt{R^{2}+\rho^{2}-2 R \rho \cos (\phi-\theta)} \cdot \\
& \cdot e^{j \frac{2 \pi}{\lambda} \sqrt{R_{2}^{2}+\rho^{2}-2 R_{2} \rho \cos (\phi-\theta-\beta)}} \sqrt{R_{2}^{2}+\rho^{2}-2 R_{2} \rho \cos (\phi-\theta-\beta)} f
\end{aligned}
$$

In practice a small horn with some directivity will be used instead of an isotropic antenna, increasing the system sensitivity and reducing unwanted reflections. The taper caused by the radiation diagram in the target region sides can be compensated introducing a weighting function in the focusing operator, in order to produce a flat sensitivy across the reconstruction domain. The main drawback of the SWISAR technique is the computational load, considerably larger than in Fast Fourier Transform (FFT) based plane wave ISAR. The algorithm efficiency can be improved significantly taking in account that (2) contains a convolution in $\phi$, which can be calculated in the spectral domain with FFT techniques. Moreover if a fixed measurement setup is adopted, the focusing operator $\xi$ can be calculated once and stored in a file to be used in the whole campaign of reconstructions. In this case the CPU time for a typical reconstruction of a $40 \mathrm{~cm}$ scaled-down model at $\mathrm{K}$ band is around $15^{\prime}$ on a HP9000-370 workstation.

Results

Fig. 2 shows the reconstruction of numerically simulated 5 point-like objects placed on a radial line for a frequency range of 18 to $26 \mathrm{GHz}$ and an angular scan of $360^{\circ}$. The reconstruction domain is a circle of diameter $0.46 \mathrm{~m}$, the horn antenna is placed at a distance $\mathrm{R}=0.6 \mathrm{~m}$ from the Origin. The lateral taper of the field radiated in the target area has been compensated. Note that the responses are invariant in shape and level with the scatterers position, also the spatial resolution and sidelobes are comparable to those obtained with plane wave ISAR. This interesting qualitites are not maintained if the antenna is placed very close to the target. From numerical simulations it has been found that invariance and plane wave similar response apply in SWISAR under the following conditions:

- The frequency step of the measurement does not exceed the Nyquist sampling 
criterion, usual in plane wave ISAR to prevent spatial domain aliasing.

- The distance of the antenna with respect to the target rotation axis is $R \geq 1.5 \mathrm{D}$, being $\mathrm{D}$ the diameter of the reconstruction spatial domain (target extension). In practice this condition is not very restrictive, specially if we compare it with the far-field condition $R \geq 2 D^{2} / \lambda$.

To validate experimentally the SWISAR algorithm several measurements have been performed substituting a small compact range installed in an anechoic chamber [3] by two simple standard gain $\mathrm{K}$ band horn antennas placed side to side in $\mathrm{H}$ polarization. The target was placed on a low reflectivity foam rotative support at $1 \mathrm{~m}$ distance of the antennas. The reconstructed region was a circle of $40 \mathrm{~cm}$ in diameter. The system was calibrated following the usual compact range methodology: empty chamber subtraction, reference target normalization and time domain quiet zone windowing. A $7 \mathrm{~cm}$ diameter metallic sphere was the reference target of well known RCS. Fig. 3 shows the reflectivity image of a metallic $228 \times 45 \mathrm{~mm}$ cylinder with flat ends processing $18-26 \mathrm{GHz} / 360^{\circ}$ data. Fig.4 corresponds to a fighter scaled-down metallized piane $34 \mathrm{~cm}$ long, the image shows the high reflectivity of the flat rear fuselage sides. These results are nearly identical to those previously obtained with a compact range working in the same band and using a conventional ISAR algorithm [3].

Conclusions

An spherical wave SWISAR imaging algorithm has been presented and evaluated. Although the formulation and implementation are more complicated and numerically demanding compared to conventional plane wave ISAR, the experimental setup is considerably simplified allowing to account for bistatic gemoetries and taper of the illumination. The results presented are similar to those obtained with plane wave illumination. A 2D formulation has been used which is strictly valid for bodies which satisfy far-field conditions in the height (z) dimension as easily occurs with aircraft. The possibility of obtaining RCS data from the spherical wave near field measurements is presently investigated.

Acknowledgement

This work has been sponsored by the Spanish Science and Tecnology Commission (CICYT TIC 88-288E).

References

[1] D.L.Mensa "High resoution radar imaging", Artech House, Dedham, 1982.

[2] X.Fábregas, A.Broquetas, L.Jofre, "Selective identification of radar target scatterers using ISAR techniques", Proc. of the II International Conf. on Electromagnetics in Aerospace Applications, pp.33-36, Torino (Italy), Sept. 1991.

[3] A.Broquetas, X.Fábregas, J.Xarrié, L.Jofre, A.Cardama, "A Compact system for RCS measurement and imaging up to $40 \mathrm{GHz}$, Proceedings de Journées Internationales de Nice sur les Antennes (JINA-90)s), pp.596-599, Nice 13-15 Nov. 1990. 


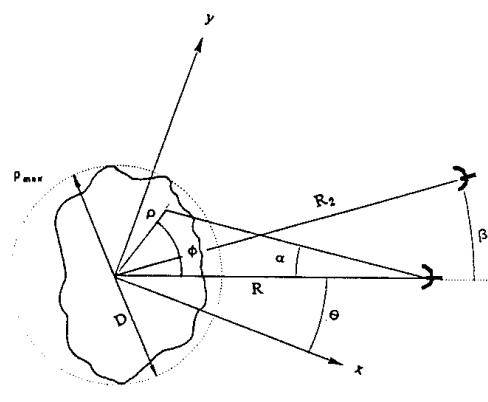

Fig. 1 Measurement geometry

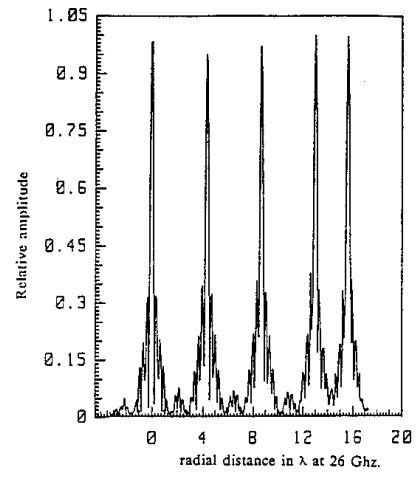

Fig.2 Reconstruction of five scatterers radially aligned

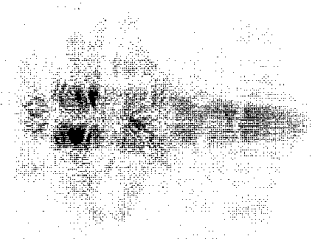

Fig. 4 Image of a plane model obtained at $K$ Band $/ 360^{\circ}$
Fig. 3 Image of a metallic cylinder measured at $K$ Band $/ 360^{\circ}$ 